\title{
DRAM1 regulates the migration and invasion of hepatoblastoma cells via autophagy-EMT pathway
}

\author{
CHAO CHEN $^{1}$, QING-YU LIANG ${ }^{2}$, HUI-KANG CHEN ${ }^{1}$, PIN-FEI WU ${ }^{1}$, ZHEN-YU FENG $^{3}$, \\ XIAO-MING MA ${ }^{3}$, HAO-RONG WU ${ }^{3}$ and GUO-QIANG ZHOU ${ }^{1}$ \\ ${ }^{1}$ Department of General Surgery, Changshu Second People's Hospital, The Fifth Hospital \\ Affiliated to Yangzhou University, Changshu, Jiangsu 215500; ${ }^{2}$ Department of General Surgery, \\ The First Hospital of Zhang Jia Gang, Zhangjiagang, Jiangsu 215600; ${ }^{3}$ Department of General Surgery, \\ The Second Hospital Affiliated to Suzhou University, Suzhou, Jiangsu 215004, P.R. China
}

Received September 19, 2017; Accepted May 3, 2018

DOI: $10.3892 / \mathrm{ol} .2018 .8937$

\begin{abstract}
DNA-damage regulated autophagy modulator 1 (DRAM1) is known as a target of TP53-mediated autophagy, and has been reported to promote the migration and invasion abilities of glioblastoma stem cells. However, the precise contribution of DRAM1 to cancer cell invasion and migration, and the underlying mechanisms remain unclear. In the present study, small interfering (si)RNA or short hairpin RNA mediated knockdown of DRAM1 was performed in hepatoblastoma cells and the migration and invasion abilities were detected in vitro and in vivo. To investigate the underlying mechanisms, western blotting and immunofluorescence were used to detect the expression of autophagy-associated proteins and epithelial-mesenchymal-transition (EMT)-associated markers. The results showed that DRAM1 knockdown by specific siRNA abrogated cell autophagy, as well as inhibited the migration and invasion of HepG2 cells in Transwell assays, which may be reversed by rapamycin treatment. In addition, DRAM1 knockdown increased the expression of E-Cadherin while decreased the expression of vimentin in HepG2 cells, which was also be reversed by rapamycin treatment. Taken together, these results suggest that DRAM1 is involved in the regulation of the migration and invasion of HepG2 cells via autophagy-EMT pathway.
\end{abstract}

\section{Introduction}

Hepatoblastoma (HB) is one of the most common solid tumors, which caused cancer-associated mortality worldwide (1). As

Correspondence to: Dr Guo-Qiang Zhou, Department of General Surgery, Changshu Second People's Hospital, The Fifth Hospital Affiliated to Yangzhou University, 68 Haiyunanlu Road, Changshu, Jiangsu 215500, P.R. China

E-mail: chowgq@sina.com

Key words: DRAM1, autophagy, migration, invasion, epithelial-mesenchymal-transition the most common malignant liver tumor of childhood, the incidence peaks in the first two years of life, with the majority of cases presenting by 5 years of age. Slight male preponderance is observed. HB may be present at birth and prenatal cases have been reported $(2,3)$. The molecular pathogensis of $\mathrm{HB}$ is remained to be elucidated. Different molecular alterations have been identified as being involved in the genesis of HB. Particularly, a deregulation of different signaling pathways has been described among which Wnt signaling (4-6), Sonic Hedgehog (7), Notch and phosphatidylinositol 3-kinase/protein kinase $\mathrm{B} / \mathrm{mammalian}$ target of rapamycin are counted as the main players $(8,9)$. It is of great importance to understand the molecular mechanisms to explore more targeted therapeutic strategies. HepG2 cells were isolated from a human liver biopsy of a 15-year old male with HB, which were shown to be a HB cell line (10-12).

DNA-damage regulated autophagy modulator 1 (DRAM1) is an evolutionarily conserved transmembrane protein, which localizes predominantly to lysosomes and acts as a target of tumor protein p53 (TP53)-mediated autophagy and programmed cell death (13). Recent reports have demonstrated that high levels of DRAM1 were associated with shorter overall survival in glioblastoma multiforme (GBM) patients, and knockdown of DRAM1 inhibited the migration and invasion abilities of glioblastoma stem cells (GSCs) (14). However, the precise contribution of DRAM1 to cancer cell invasion and migration and the underlying mechanisms remain unclear. Autophagy is an evolutionally conserved process in which amino acids, nutrients, and lipids are recycled when cells go through nutrient and oxygen deprivations (15). DRAM1 acts as a regulator of autophagy mediated by p53 in response to genotoxic stresses through regulation of the clearance of autophagosomes by promoting lysosomal acidification and inducing the activation of lysosomal enzymes (16).

Epithelial-mesenchymal-transition (EMT) represents a process of fast changes, during which the cell phenotype changes from epithelial to mesenchymal, the expression of mesenchymal markers were upregulated, the actin cytoskeleton was reorganization, cell-cell adhesion structures were destructed, and pseudopod formation emerges (17). During the EMT, the migratory ability of epithelial cells is enhanced (17). 
Autophagy is a catabolic process that mediates degradation of unnecessary or dysfunctional cellular components $(18,19)$. These two important processes in cancer are linked in an intricate relationship. EMT requires autophagy to support viability of potentially metastatic cancer cells, while a number of additional evidence indicates that autophagy acts to prevent EMT and that the activation of the autophagic machinery may determine reversion of the EMT phenotype in cancer cells (20-24).

This study was designed to investigate the effect of DRAM1 on cell invasion and migration, as well as explore the underlying mechanisms involved in cell autophagy and EMT. We provided the evidence that DRAM1 knockdown inhibited cell invasion and migration abilities of HB cells by inhibiting the autophagy-EMT pathway.

\section{Materials and methods}

Antibodies and reagents. Antibodies for E-Cadherin and Vimentin were purchased from Cell Signaling Technology, Inc. (Danvers, MA, USA). Antibodies for DRAM1, P62 and LC3 were purchased from Abcam (Cambridge, MA, USA). Antibody for $\beta$-actin was purchased from Santa Cruz Biotechnology, Inc. (Dallas, TX, USA). Rapamycin was purchased from Sigma-Aldrich (Merck KGaA, Darmstadt, Germany). Transwell chamber was purchased from Corning Incorporated (Corning, NY, USA). Matrigel was purchased from BD Biosciences (Franklin Lakes, NJ, USA).

Cell culture. Human HB derived HepG2 cells were obtained from the American Type Culture Collection and cultured in Dulbecco's modified Eagle's medium (DMEM; 11965500; Gibco; Thermo Fisher Scientific, Inc., Waltham, MA, USA) containing $10 \%$ fetal bovine serum (FBS; 086150008; Wisten Inc., Anthem, AZ, USA), $100 \mathrm{IU} / \mathrm{ml}$ penicillin, and $100 \mathrm{IU} / \mathrm{ml}$ streptomycin in a humidified incubator at $37^{\circ} \mathrm{C}$ under $5 \% \mathrm{CO}_{2}$ atmosphere, and passaged at preconfluent densities using $0.25 \%$ trypsin solution every 2 to 3 days. Cells were stored and used within 3 months after resuscitation from cryopreservation status.

Transfection and RNA interference. The DRAM1 shRNA (shDRAM1: Tracking number TRCN0000161451, clone ID NM 018370.1-1356s1c1) were purchased from Sigma-Aldrich and the small interfering (si)RNA targeting DRAM1 was purchased from Shanghai GenePharma Co., Ltd. (Shanghai, China). For establish stable DRAM1 knockdown cells, HepG2 cells were transfected with DRAM1 shRNA and were selected in cell culture medium containing $1.5 \mathrm{mg} / \mathrm{ml}$ poromycin for 1 week. Cells were then cultured in culture medium for in vivo experiments. For transfection, cells were plated in 6-well plates at $30 \%$ confluency, and siRNA duplexes were introduced into the cells using Lipofectamine 2000 (Invitrogen; Thermo Fisher Scientific, Inc.) according to the manufacturer's recommendation. The siRNAs targeting sense sequences were as follows: DRAM1-1, 5'-CCACGAUGUAUACAA GAUA-3'; DRAM1-2, 5'-CCACGAAAUCAAUGGUGA-3'; Negative control, 5'-UAAGGCUAUGAAGAGAUAC-3'. The knockdown efficiency of specific proteins was determined by western blot analysis $48 \mathrm{~h}$ after shRNA or siRNA treatment.
Short hairpin RNA (shRNA) lentiviruses production and transduction. A shRNA against DRAM1 was cloned into the pLKO.1 vector according to the manufacturer's protocol (Addgene, Inc., Cambridge, MA, USA). pLKO.1, scrambled shRNA (negative control), pMD2.G (used for viruspackaging) and psPAX2 (used for virus packaging) were purchased from Addgene, Inc. All constructs were verifed by sequencing. Lentiviruses were produced by co-transfecting 293FT cells (Invitrogen; Thermo Fisher Scientifc, Inc.) in 10-cm dishes with $10 \mu \mathrm{g}$ pLKO.1-shRNA, $2.5 \mu \mathrm{g}$ pMD2.G and $7.5 \mu \mathrm{g}$ psPAX2 using Lipofectamine 2000 (Invitrogen; Thermo Fisher Scientific, Inc.). HepG2 cells were infected with DRAM1 shRNA. Virus-containing medium was removed after $16 \mathrm{~h}$ and replaced with fresh DMEM. After $24 \mathrm{~h}$, the cells were used experimentally.

Transwell assay. The migration and invasion abilities were detected using Transwell chambers with $8 \mu \mathrm{m}$ pore filters. The filters used for invasion assays were coated with $30 \mu \mathrm{l}$ pre-diluted Matrigel (diluted at a ratio of 1:6 with serum-free DMEM medium). Cells (1x10 $10^{5}$ cell/well for migration assay, $2.5 \times 10^{5}$ cell/well for invasion assay) were appropriately added to the upper chambers. And then $0.6 \mathrm{ml}$ DMEM medium supplemented with $10 \%$ FBS was added to the lower chambers as a chemoattractant. After incubation for $24 \mathrm{~h}$, migrated or invaded cells were stained with DAPI. The cell number was counted under an optical microscope (5 different visual fields were randomly selected for each membrane and observed under x600 magnification lenses). All experiments were performed at least in triplicate.

Western blot analysis. Protein was extracted from cells using cell lysis solutions containing protease inhibitors and phosphorylase inhibitors. Equal amounts of protein were fractionated on Tris-glycine SDS-polyacrylamide gels and subjected to electrophoresis and transferred to NC membranes. Membranes were blocked with 5\% non-fat milk with Tris buffered Saline-Tween-20 (TBS-T), and then incubated with primary antibodies against DRAM1, P62, LC3, E-Cadherin, or Vimentin. After washing in TBST, membranes were incubated with fluorescent secondary antibodies. $\beta$-actin was used as the loading control. Immunoreactivity was detected using ODYSSEY INFRARED IMAGER (Li-COR Biosciences, Nebraska, NE, USA). The signal intensity of primary antibody binding was quantitatively analyzed with Image $\mathrm{J}$ software (W.S. Rasband, Image J; National Institutes of Health, Bethesda, MD, USA).

Immunofluorescence. The HepG2 cells were seeded onto cover glass in 24 well plates and cultured to the appropriate confluency. Thereafter, cells were washed with phosphate-buffered saline (PBS) for $5 \mathrm{~min} x 3$ times. Then cells were treated with precooled alcohol for $15 \mathrm{~min}$ before blocked in PBS containing 1\% BSA and 0.1\% Triton X-100 for $1 \mathrm{~h}$ at room temperature. Then the cells were incubated with primary antibody overnight at $4^{\circ} \mathrm{C}$. After washed 3 times with PBS for $10 \mathrm{~min}$, the cells were incubated with Cy3-conjugated donkey anti-rabbit $\mathrm{IgG}$ for $1 \mathrm{~h}$ at room temperature. After another $10 \mathrm{~min} \times 3$ times of washing with 
A
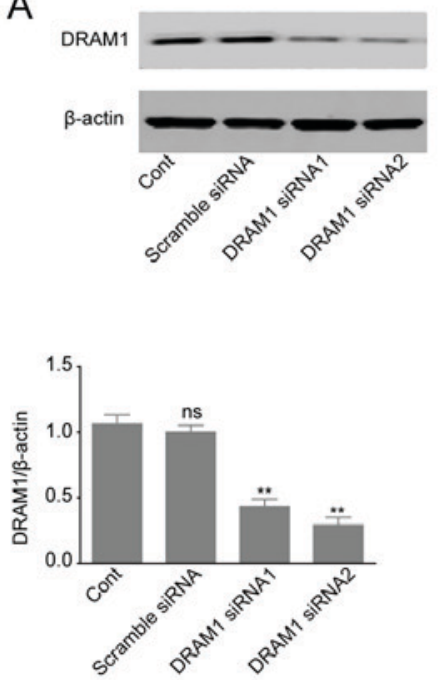

E

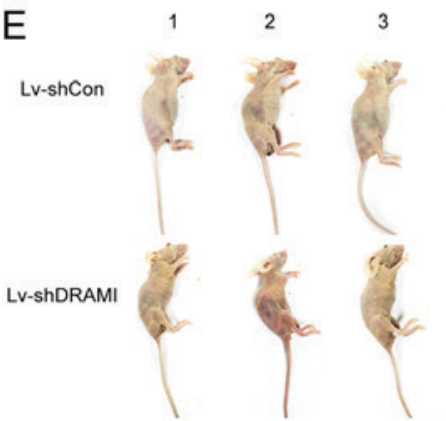

$\mathrm{B}$
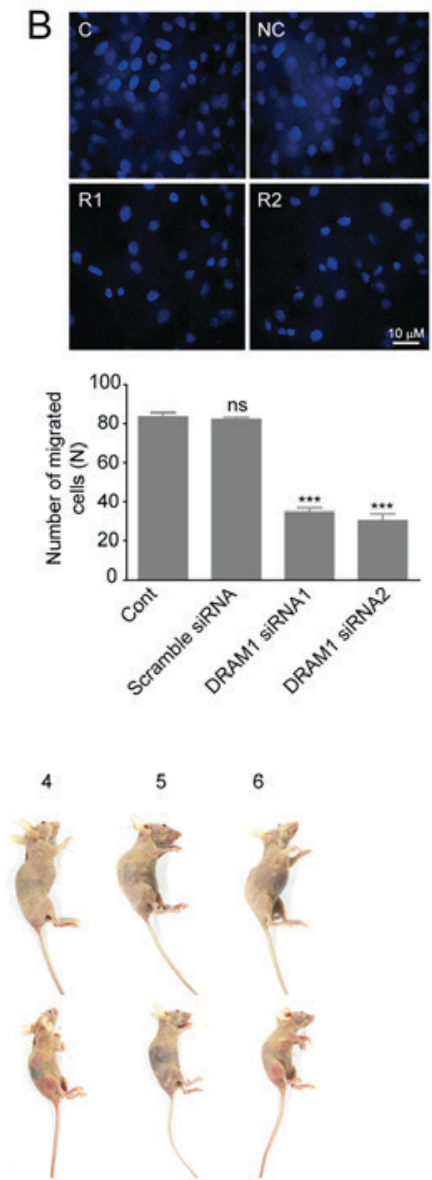

C
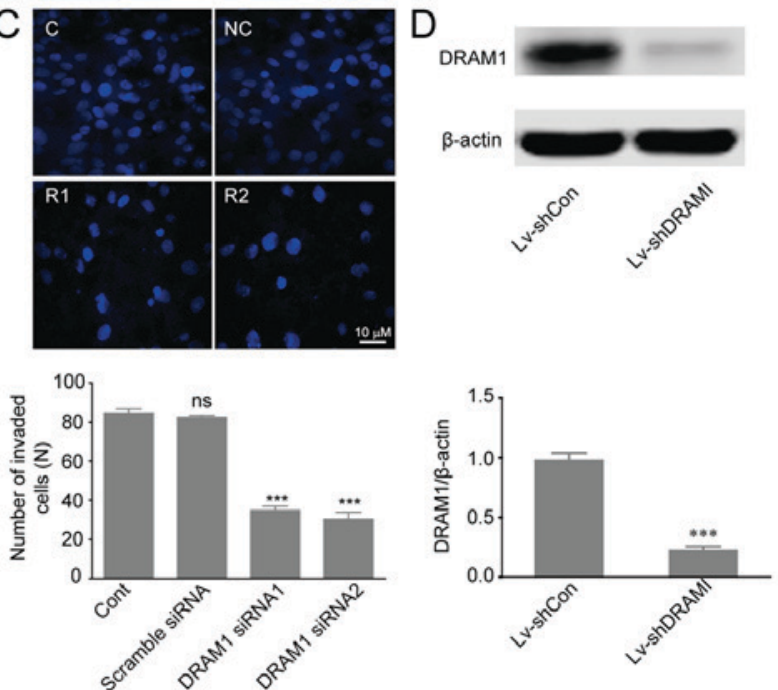

$\mathrm{F}$

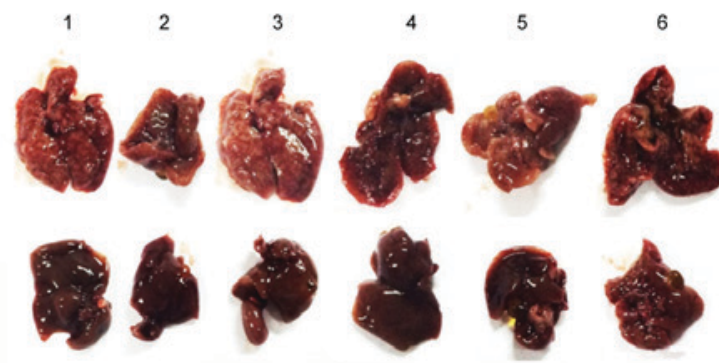

Figure 1. Knockdown of DRAM1 inhibited invasion and metastasis of HepG2 cells in vivo and in vitro. (A) Knockdown efficiency of DRAM1 in HepG2 cells. Transient transfection of siRNAs was applied to knockdown DRAM1 expression for $48 \mathrm{~h}$. The protein levels of DRAM1 were detected with Western blotting. $\beta$-actin protein was used as a loading control. Quantitative analysis was performed with Image J. (B) Migration and (C) invasion assay of HepG2 cells with or without DRAM1 siRNA. Invasion assay was performed with transwell-inserts coated with Matrigel. Images were taken with a microscope (magnification, $\mathrm{x} 600$ ). Values were means \pm SEM from 3 independent experiments. ${ }^{* *} \mathrm{P}<0.01 ;{ }^{* * * *} \mathrm{P}<0.001 ; n s, \mathrm{P}>0.05$ vs. control group. (D) Lentivirus-mediated shRNA decreased DRAM1 expression in HepG2 cells. Analysis of DRAM1 knockdown efficiency in HepG2 cells. Values were means \pm SD from 3 independent experiments. ${ }^{* * *} \mathrm{P}<0.001$, compared with Lv-shCon. (E) In vivo tumor metastasis assay of DRAM1 knockdown cells. HepG2 cells were intravenously injected into the tail vein of nude mice for 4 weeks. (F) The mice were sacrificed and examined for tumor metastases. DRAM1, DNA-damage regulated autophagy modulator 1; SD, standard deviation; SEM, standard error of the mean.

PBS, cells were incubated with DAPI for $10 \mathrm{~min}$, and dehydrated in increasing grades of ethanol and cover-slipped with Fluoromount Aqueous Mounting Medium (Sigma F4680; Sigma-Aldrich; Merck KGaA). The slices were analyzed with a laser scanning confocal unit (Zeiss LSM 710; Carl Zeiss, Jena, Germany).

In vivo tumor growth analysis. The control shRNA-transfected cells and DRAM1 shRNA-transfected cells $\left(1 \times 10^{7}\right)$ were intravenously injected into the tail vein of 6-week-old female athymic nude mice (Shanghai SLAC Laboratory Animal Co. Ltd., Shanghai, China). At 4 weeks later, the mice were anesthetized and photographed. After the mice were sacrificed, the livers were removed and photographed. All animal procedures were approved and monitored by the local Animal Care and Use Committee in Soochow University (License no. Syxk; $\mathrm{Su}-0062)$.

Statistical analysis. All data were presented as means \pm SEM. Data were subjected to one-way ANOVA using the GraphPad Prism software statistical package (GraphPad Software;
GraphPad Software, Inc., La Jolla, CA, USA). When a significant group effect was found, post hoc comparisons were performed using the Student-Newman-Keuls test to examine special group differences. Independent group t-tests were used for comparing two groups. Significant differences with $\mathrm{P}<0.05,0.01$, and 0.001 are indicated by ${ }^{*},{ }^{* *},{ }^{* * *}$, respectively. All calculations were performed using the 14.0 SPSS software package (SPSS, Inc., Chicago, IL, USA).

\section{Results}

DRAM1 knockdown inhibits the invasion and metastasis of Hep 2 cells in vivo and in vitro. Previous studies reported that high expression of DRAM1 indicated poor prognosis in GBM tumors, and that inhibition of DRAM1 expression reduced invasion and metastasis of GSCs $(14,25)$. In order to further investigate the effects of DRAM1 on invasion and metastasis of HepG2 cells, we designed two different DRAM1 siRNA molecules (DRAM1 siRNA1, DRAM1 siRNA2) to knockdown DRAM1 expression. HepG2 cells were transfected with scramble siRNA, DRAM1 siRNA1 or DRAM1 siRNA2 at 
A

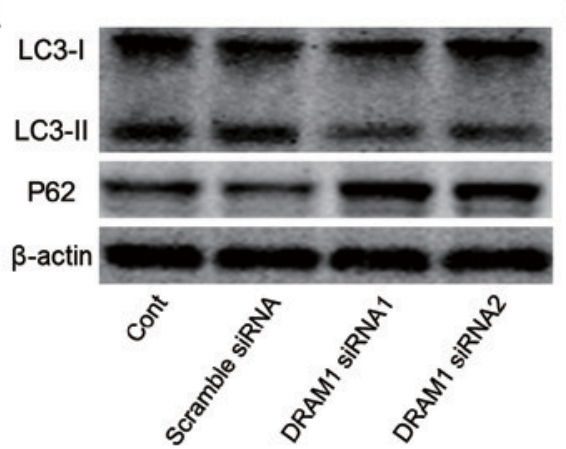

B

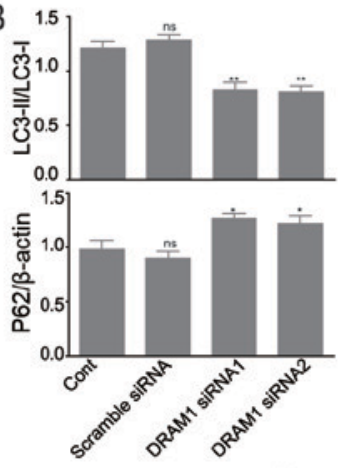

$\mathrm{E}$

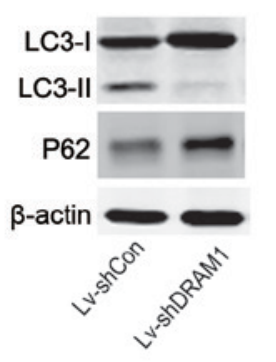

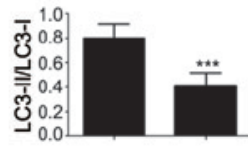

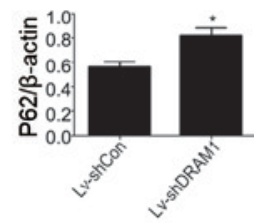

C

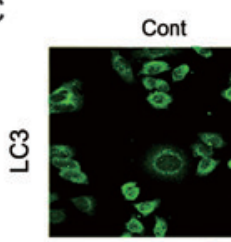

Scramble siRNA
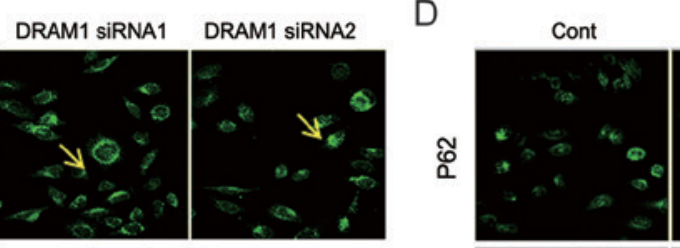

Scramble siRNA
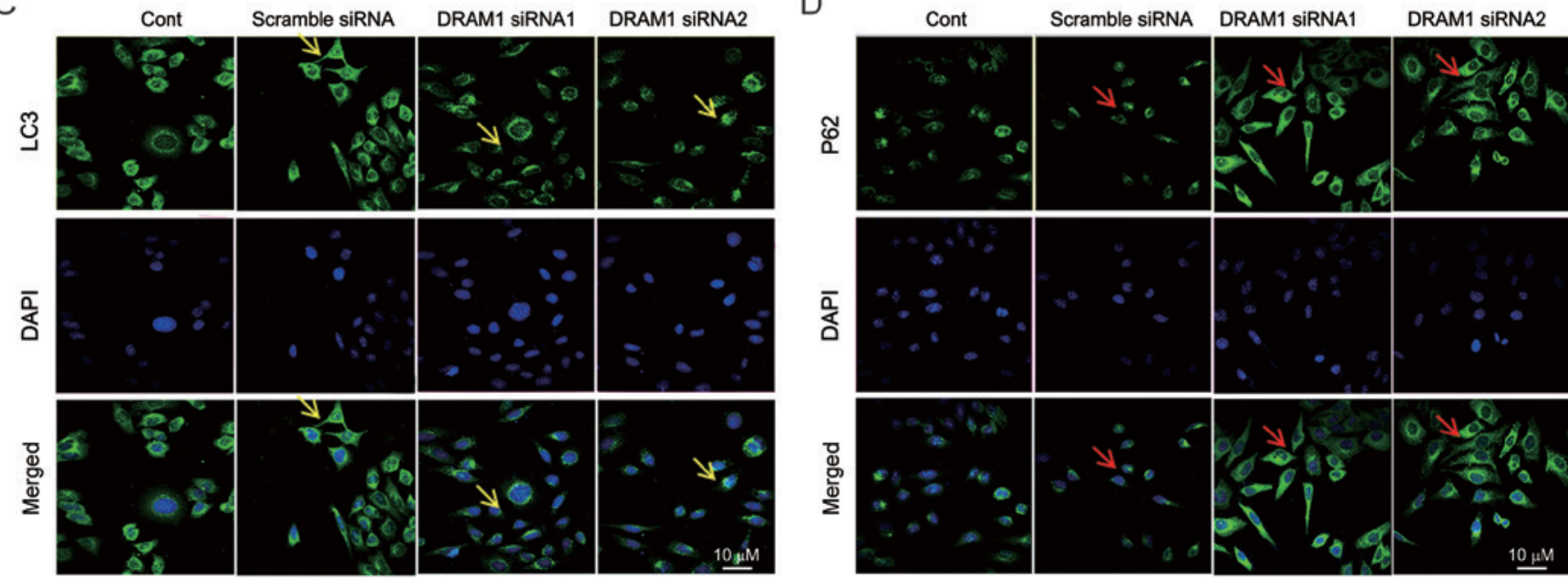

Figure 2. Knockdown of DRAM1 inhibited autophagy in HepG2 cells. (A and B) Western blot analysis of autophagy related protein LC3I, LC3II and p62 in HepG2 cells, in response to knockdown of DRAM1. (C and D) Immunofluorescence analysis of LC3II and p62 in HepG2 cells, in response to knockdown of DRAM1. Values are means \pm SEM from 3 independent experiments. ${ }^{*} \mathrm{P}<0.05 ;{ }^{* *} \mathrm{P}<0.01 ; \mathrm{ns}, \mathrm{P}>0.05$ vs. control group. LC 3 fluorescence signal: Yellow arrow; 62 fluorescence signal: Red arrow (E) HepG2 cells were transduced with lentiviruses expressing a scramble oligo and a sequence targeting DRAM1 (shDRAM1). Western blot analysis of autophagy related protein LC3I, LC3II and p62 in HepG2 cells, in response to knockdown of DRAM1. Values are means \pm SEM from 3 independent experiments. ${ }^{*} \mathrm{P}<0.05 ;{ }^{* * *} \mathrm{P}<0.001$. DRAM1, DNA-damage regulated autophagy modulator 1 ; SEM, standard error of the mean.

the concentration of $80 \mathrm{nM}$ for $48 \mathrm{~h}$. Western blot analysis of DRAM1 protein levels showed 57 and $71 \%$ of silence efficiency with DRAM1 siRNA1 and DRAM1 siRNA2, respectively (Fig. 1A). Transwell chambers for migratory and invasive culture system were used to detect the migration and invasion abilities of HepG2 cells after DRAM1 was knocked down. The results showed that knockdown of DRAM1 significantly reduced the number of migrated (Fig. 1B) and invaded (Fig. 1C) cells, which indicated that DRAM1 knockdown inhibited migration and invasion abilities of HepG2 cells. Moreover, we transduced HepG2 cells with vectors expressing shRNAs against DRAM1 or the scramble shRNA (Fig. 1D) and grafted these cells into nude mice by tail vein injection ( $1 \times 10^{7}$ cells per nude mouse). 4 weeks later the DRAM1 knockdown cells exhibited slower growth and lower metastasis compared to the control shRNA-transfected cells (Fig. 1E and F). Collectively, these results suggested that knockdown of DRAM1 inhibited the migration and invasion abilities of HepG2 cells both in vitro and in vivo.

DRAM1 knockdown inhibits autophagy in HepG2 cells. In order to investigate the mechanisms underlying the inhibitory effects of DRAM1 knockdown on cell invasion and metastasis, the levels of autophagy-related proteins were detected. As shown in Fig. 2A and B, decreased transformations of
LC3I to LC3II as well as increased expressions of p62 were observed in DRAM1 siRNA groups, indicating that autophagy was inhibited by DRAM1 knockdown (Fig. 2A and B). We next performed immunofluorescence to further detect the distribution of LC3 and p62 in HepG2 cells. In consistent with Western blot analysis, the results showed decreased distribution of LC3II and increased expression of p62 in the cytoplasm in DRAM1 knockdown cells (Fig. 2C and D), which suggested that DRAM1 knockdown inhibited cell autophagy. Also, similar result was observed in knockdown cells by shDRAM1 (Fig. 2E).

DRAM1 knockdown abrogates RAPA-induced autophagy in HepG2 cells. We continued to verify the effects of DRAM1 knockdown on cell autophagy by applying RAPA, an autophagy inducer. HepG2 cells were transfected with DRAM1 siRNAs for $48 \mathrm{~h}$ with or without RAPA treatment and autophagy related protein levels were detected by Western blots. The results showed that the autophagy induced by RAPA stimulation was inhibited by DRAM1 knockdown. RAPA treatment caused an increased transformation of LC3I to LC3II and a reduced expression of p62 (Fig. 3A and B), and the stimulatory effects of RAPA on cell autophagy were obviously inhibited when DRAM1 was knocked down. These results further verified the inhibitory potential of DRAM1 knockdown on cell autophagy. 
A

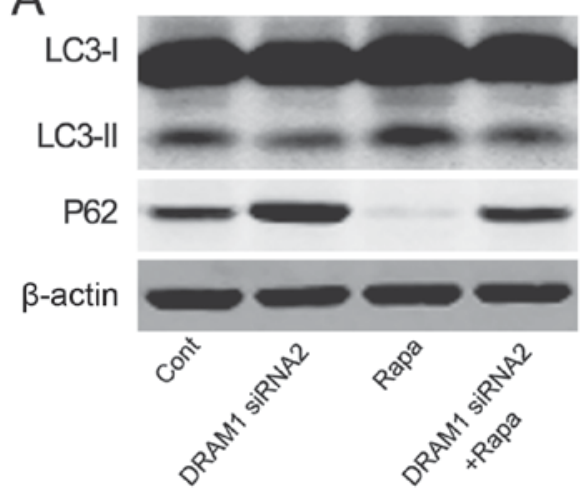

B

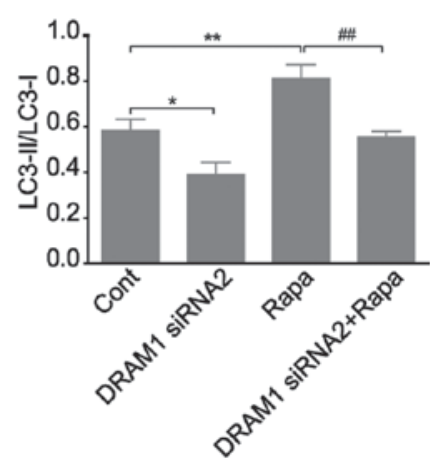

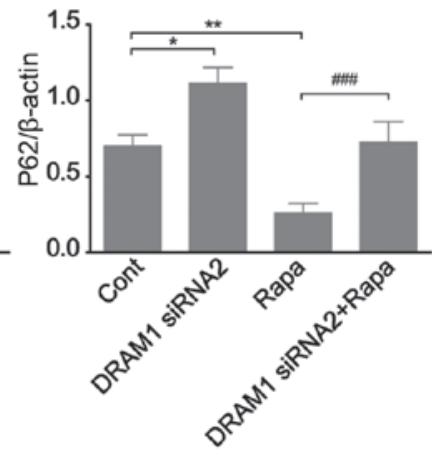

Figure 3. DRAM1 knockdown abrogates RAPA-induced autophagy in HepG2 cells. HepG2 cells treated with $200 \mathrm{nM}$ RAPA for $12 \mathrm{~h}$. (A and B) Western blot analysis of the autophagy related protein. Values were means \pm SEM from 3 independent experiments. ${ }^{*} \mathrm{P}<0.05 ;{ }^{* *} \mathrm{P}<0.01$ vs. control group, ${ }^{\# \#} \mathrm{P}<0.01 ;{ }^{\# \# *} \mathrm{P}<0.001$ vs. RAPA. DRAM1, DNA-damage regulated autophagy modulator 1; SEM, standard error of the mean.

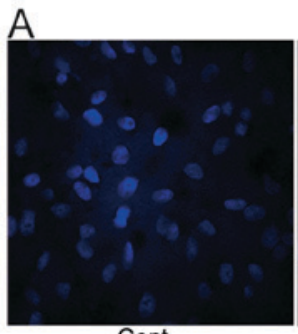

B

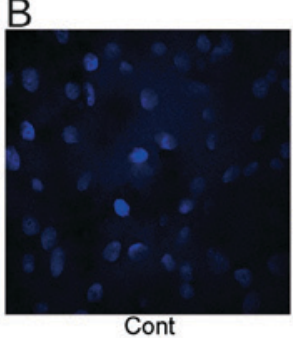

C

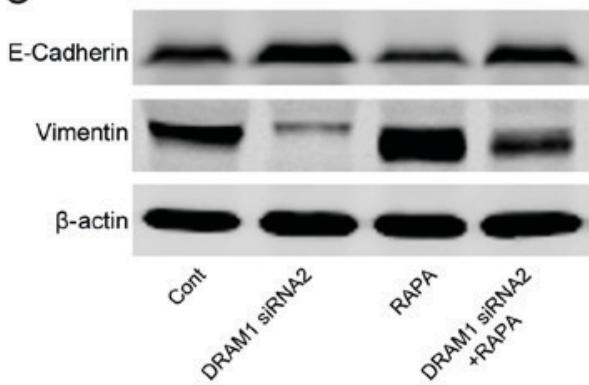

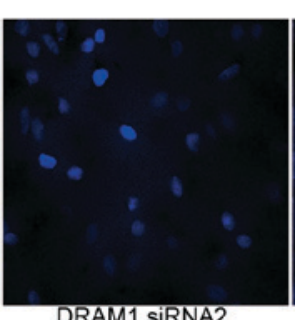

DRAM1 SIRNA2

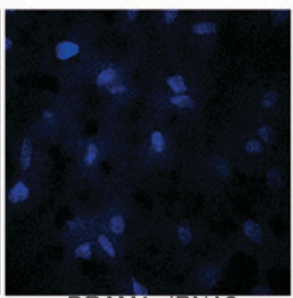

DRAM1 SIRNA2

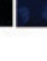

.

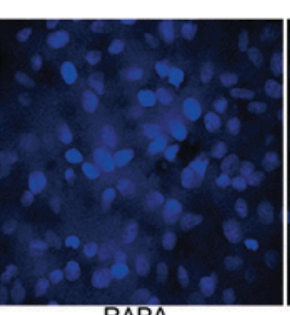

RAPA
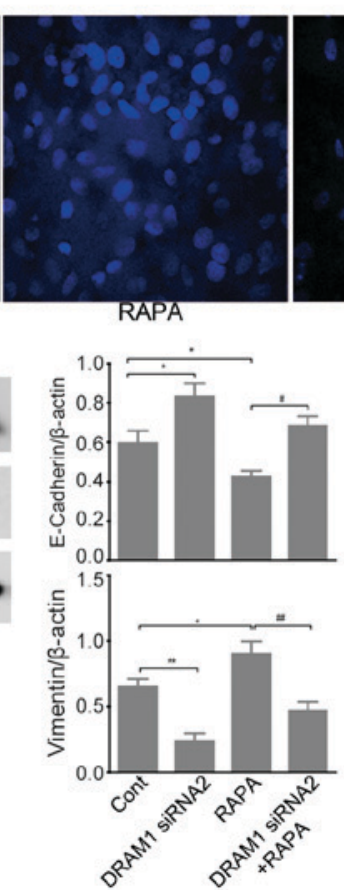

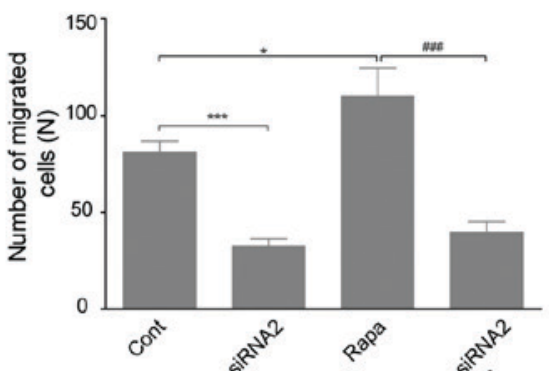

DRAM1 STRNA2

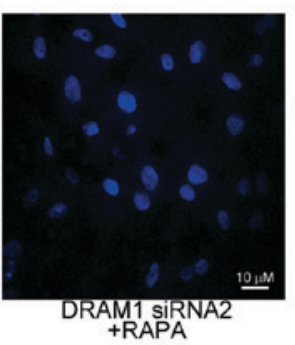

$\mathrm{D}$

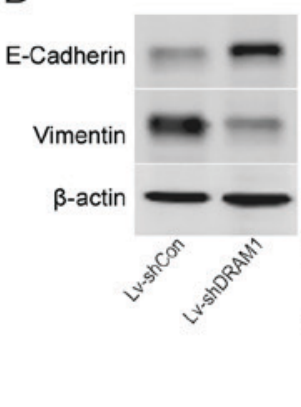

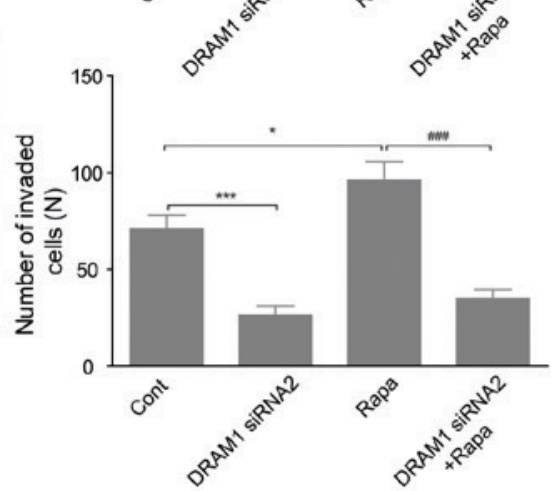

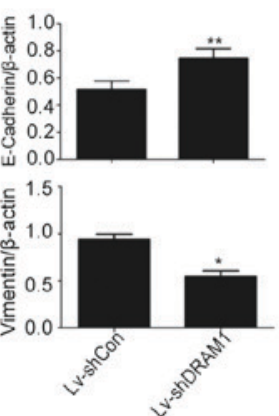

Figure 4. DRAM1 knockdown inhibits invasion and migration of HepG2 cells. (A and B) Migration and invasion assay of HepG2 cells with or without DRAM1 siRNA. Invasion assay was performed with transwell-inserts coated with Matrigel. Images were taken with a microscope (magnification, $\mathrm{x} 600$ ), ${ }^{*} \mathrm{P}<0.05 ;{ }^{* * *} \mathrm{P}<0.001$ vs. control; ${ }^{\# \#} \mathrm{P}<0.001$ vs. RAPA. (C) Western blot analysis of E-cadherin, and vimentin in HepG2 cells transfected with DRAM1 siRNA. Values were means \pm SEM from 3 independent experiments. ${ }^{*} \mathrm{P}<0.05 ;{ }^{* *} \mathrm{P}<0.01$ vs. control group; ${ }^{\#} \mathrm{P}<0.05$; ${ }^{\# \#} \mathrm{P}<0.01$ vs. RAPA. (D) In vivo tumor metastasis assay of DRAM1 knockdown cells. HepG2 cells were intravenously injected into the tail vein of nude mice for 4 weeks. The mice were sacrificed and their livers were harvested. Liver specimens were examined for the protein levels of E-cadherin and Vimentin by western blotting. Values were means \pm SEM from 3 independent experiments. ${ }^{*} \mathrm{P}<0.05 ;{ }^{* *} \mathrm{P}<0.01$ vs. control group. DRAM1, DNA-damage regulated autophagy modulator 1 ; SEM, standard error of the mean.

Invasion and migration of HepG2 cells were inhibited through autophagy-EMT pathway when DRAMI was knocked down. We next set out to discover the mechanisms underlying the inhibitory effects of DRAM1 knockdown on the cell invasion and metastasis. Transwell chamber migratory and invasive culture systems were adopted to detect the migratory and 
invasive ability of HepG2 cells after DRAM1 was knocked down. As showed in Fig. 4A and B, cell invasion and metastasis were inhibited after DRAM1 knockdown. Moreover, the stimulatory effects of RAPA on cell invasion and metastasis were also obviously rescued by DRAM1 knockdown (Fig. 4A and B).

Since we have observed significant inhibition of DRAM1 knockdown on the invasion and metastasis abilities of HepG2 cells both in vitro and in vivo, we next detected the expression of EMT related proteins by western blots. The results showed an increased expression of E-cadherin and a decreased expression of vimentin in DRAM1 knockdown HepG2 cells, which indicated an inhibitory effect of DRAM1 knockdown on EMT. On the contrary, RAPA treatment caused a decreased expression of E-cadherin and an increased expression of vimentin, both of which were significantly reversed by the combined treatment of DRAM1 siRNA (Fig. 4C). Similar result was obtained by shDRAM1 (Fig. 4D). Collectively, these results suggested that the inhibitory effects of DRAM1 knockdown on the invasion and migration abilities of HepG2 cells likely worked through the autophagy-EMT pathway.

\section{Discussion}

HepG2 cells were shown to be a HB cell line, which did not affected the outcomes of the study. HB is the most common liver malignant tumor diagnosed by the age of 4 years, accounting for $80 \%$ of liver cancers in children under the age of 15 years, among patients with localized $\mathrm{HB}$, surgical resection is a common treatment option $(26,27)$. Liver transplantation is considered to be the only curative therapy, however, the majority of patients with advanced HB are not suitable for transplantation (1). Therefore, a more comprehensive understanding of the regulatory mechanisms of HB invasion and migration is beneficial for the survival improvement of HB patients. Previous reports have shown that DRAM1 played an important role in the migration and invasion of GSCs (14). Our previous results also revealed that DRAM1 was highly expressed in intestinal cancer. However, little is known about the precise contribution of DRAM1 in the migration and invasion of HB cells. In this study, we found that DRAM1 knockdown could inhibit the migration and invasion of HepG2 cells in the setting of transwell assay, which was further confirmed by animal experiments.

EMT is a natural process in which epithelial cells obtain characteristics of mesenchymal cells, which is recognized as an important procedure in cell invasion and metastasis. EMT happens in normal cellular processes, however, it may also be exploited by cancer cells, which triggers them to invade and form metastases at distant sites. EMT was reported to motivate the expression of mesenchymal markers in epithelial cells (28). The molecular regulators involved in EMT include specific molecules which distinguish epithelial cells from mesenchymal cells, and other constituents that can drive cells towards the targeted sites. Cells undergoing EMT typically show an increase in the expression of vimentin, fibronectin and integrin $\alpha v \beta 6$, as well as a decrease in the expression of E-cadherin and cytokeratins. In our present study, with the application of rapamycin, the expression of vimentin was significantly upregulated while E-cadherin was downregulated in HepG2 cells. Meanwhile, the concomitant knockdown of DRAM1 reversed the alterations of these EMT markers. Our results suggested that DRAM1 knockdown regulated EMT thus inhibiting the invasion and metastasis of HepG2 cells.

Growing evidence has suggested that autophagy is closely related to human physiology and diseases including cancers. Pervious researches revealed that DRAM1 was a lysosomal protein associated with cell autophagy $(13,29,30)$. Therefore, we speculated that autophagy may be involved in the inhibition of DRAM1 knockdown on the migration and invasion of HB cells. The results in this study demonstrated that the elevation of LC3-II and the decline of P62 stimulated by rapamycin treatment were blocked by DRAM1 knockdown, which suggested that cell autophagy was inhibited after DRAM1 knockdown. However, it is still controversial on the function of autophagy in regulating cell migration and invasion. Robin and colleagues showed that autophagy inhibition significantly reduced the invasion of tumor cells (31). Zhan et al reported that autophagy induced by the activation of toll-like receptor 3 or 4 could enhance the production of various cytokines and thereby facilitating the migration and invasion of lung cancer cells (32). On the contrary, there were also several researches suggested that autophagy could inhibit tumor cell migration and invasion $(22,33)$. In this study, we found that DRAM1 knockdown significantly decreased the levels of EMT markers and inhibited the migration and invasion of $\mathrm{HepG} 2$ cells, which could be reversed by rapamycin treatment. It needs to be clarified in future studies whether we could reverse the inhibition of migration and invasion in DRAM1-silenced HepG2 cells by activating autophagy.

In summary, DRAM1 played an important role in the regulation of HB migration and invasion. Our study demonstrated that DRAM1 knockdown inhibited cell invasion and migration by inhibiting the autophagy-EMT pathway, which could provide basic knowledge for the development of new therapies for HB in clinical practice.

\section{Acknowledgements}

Not applicable.

\section{Funding}

This work was supported by the Medicine and Technology Project For Youth Changshu (CSWSQ201707) and the National Natural Science Foundation of China (grant no. 81602613).

\section{Availability of data and materials}

The datasets used and/or analyzed during the current study are available from the corresponding author on reasonable request.

\section{Authors' contributions}

$\mathrm{CC}$ contributed to the idea conception, experimental work and manuscript preparation. QYL, HKC, PFW, ZYF, XMM and HRW contributed to the experimental work and manuscript 
preparation. GQZ guided the idea conception, experimental work and manuscript preparation. All authors reviewed the manuscript.

\section{Ethics approval and consent to participate}

Not applicable.

\section{Consent for publication}

Not applicable.

\section{Competing interests}

The authors declare that they have no competing interests.

\section{References}

1. Sia D, Villanueva A, Friedman SL and Llovet JM: Liver cancer cell of origin, molecular class, and effects on patient prognosis. Gastroenterology 152: 745-761, 2017.

2. Avni FE, Massez A and Cassart M: Tumours of the fetal body: A review. Pediatr Radiol 39: 1147-1157, 2009.

3. Catanzarite V, Hilfiker M, Daneshmand S and Willert J: Prenatal diagnosis of fetal hepatoblastoma: Case report and review of the literature. J Ultrasound Med 27: 1095-1098, 2008.

4. Apte U, Zeng G, Thompson MD, Muller P, Micsenyi A Cieply B, Kaestner KH and Monga SP: Beta-catenin is critical for early postnatal liver growth. Am J Physiol Gastrointest Liver Physiol 292: G1578-G1585, 2007.

5. Monga SP: Role and regulation of $\beta$-catenin signaling during physiological liver growth. Gene Expr 16: 51-62, 2014.

6. Tan X, Yuan Y, Zeng G, Apte U, Thompson MD, Cieply B, Stolz DB, Michalopoulos GK, Kaestner KH and Monga SP: Beta-catenin deletion in hepatoblasts disrupts hepatic morphogenesis and survival during mouse development. Hepatology 47: 1667-1679, 2008

7. Tanimizu N and Miyajima A: Notch signaling controls hepatoblast differentiation by altering the expression of liver-enriched transcription factors. J Cell Sci 117: 3165-3174, 2004.

8. Hartmann W, Küchler J, Koch A, Friedrichs N, Waha A, Endl E, Czerwitzki J, Metzger D, Steiner S, Wurst P, et al: Activation of phosphatidylinositol-3'-kinase/AKT signaling is essential in hepatoblastoma survival. Clin Cancer Res 15: 4538-4545, 2009.

9. Testa JR and Tsichlis PN: AKT signaling in normal and malignant cells. Oncogene 24: 7391-7393, 2005.

10. Aden DP, Fogel A, Plotkin S, Damjanov I and Knowles BB: Controlled synthesis of HBsAg in a differentiated human liver carcinoma-derived cell line. Nature 282: 615-616, 1979.

11. López-Terrada D, Cheung SW, Finegold MJ and Knowles BB: Hep G2 is a hepatoblastoma-derived cell line. Hum Pathol 40: $1512-1515,2009$

12. Capes-Davis A, Theodosopoulos G, Atkin I, Drexler HG, Kohara A, MacLeod RA, Masters JR, Nakamura Y, Reid YA, Reddel RR and Freshney RI: Check your cultures! A list of cross-contaminated or misidentified cell lines. Int J Cancer 127: 1-8, 2010.

13. Crighton D, Wilkinson S, O'Prey J, Syed N, Smith P, Harrison PR, Gasco M, Garrone O, Crook T and Ryan KM: DRAM, a p53-induced modulator of autophagy, is critical for apoptosis Cell 126: 121-134, 2006.

14. Galavotti S, Bartesaghi S, Faccenda D, Shaked-Rabi M, Sanzone S, McEvoy A, Dinsdale D, Condorelli F, Brandner S, Campanella M, et al: The autophagy-associated factors DRAM1 and p62 regulate cell migration and invasion in glioblastoma stem cells. Oncogene 32: 699-712, 2013.
15. Klionsky DJ and Emr SD: Autophagy as a regulated pathway of cellular degradation. Science 290: 1717-1721, 2000.

16. Zhang XD, Qi L, Wu JC and Qin ZH: DRAM1 regulates autophagy flux through lysosomes. PLoS One 8: e63245, 2013.

17. Klymkowsky MW and Savagner P: Epithelial-mesenchymal transition: A cancer researcher's conceptual friend and foe. Am J Pathol 174: 1588-1593, 2009.

18. Boya $\mathrm{P}$, Reggiori $\mathrm{F}$ and Codogno $\mathrm{P}$ : Emerging regulation and functions of autophagy. Nat Cell Biol 15: 713-720, 2013.

19. Kroemer G, Mariño G and Levine B: Autophagy and the integrated stress response. Mol Cell 40: 280-293, 2010.

20. Lv Q, Wang W, Xue J, Hua F, Mu R, Lin H, Yan J, Lv X, Chen X and $\mathrm{Hu}$ ZW: DEDD interacts with PI3KC3 to activate autophagy and attenuate epithelial-mesenchymal transition in human breast cancer. Cancer Res 72: 3238-3250, 2012.

21. Qiang L, Zhao B, Ming M, Wang N, He TC, Hwang S, Thorburn A and He YY: Regulation of cell proliferation and migration by p62 through stabilization of Twist1. Proc Natl Acad Sci USA 111: 9241-9246, 2014.

22. Catalano M, D'Alessandro G, Lepore F, Corazzari M, Caldarola S, Valacca C, Faienza F, Esposito V, Limatola C, Cecconi F and Di Bartolomeo S: Autophagy induction impairs migration and invasion by reversing EMT in glioblastoma cells. Mol Oncol 9: 1612-1625, 2015.

23. Li G, Li CX, Xia M, Ritter JK, Gehr TW, Boini K and Li PL: Enhanced epithelial-to-mesenchymal transition associated with lysosome dysfunction in podocytes: Role of p62/sequestosome 1 as a signaling hub. Cell Physiol Biochem 35: 1773-1786, 2015.

24. Gugnoni M, Sancisi V, Gandolfi G, Manzotti G, Ragazzi M, Giordano D, Tamagnini I, Tigano M, Frasoldati A, Piana S and Ciarrocchi A: Cadherin-6 promotes EMT and cancer metastasis by restraining autophagy. Oncogene 36: 667-677, 2017.

25. Humbert M, Mueller C, Fey MF and Tschan MP: Inhibition of damage-regulated autophagy modulator-1 (DRAM-1): Impairs neutrophil differentiation of NB4 APL cells. Leuk Res 36: 1552-1556, 2012.

26. Herzog CE, Andrassy RJ and Eftekhari F: Childhood cancers: Hepatoblastoma. Oncologist 5: 445-453, 2000.

27. Darbari A, Sabin KM, Shapiro CN and Schwarz KB: Epidemiology of primary hepatic malignancies in U.S. children. Hepatology 38: 560-566, 2003.

28. Xu J, Lamouille S and Derynck R: TGF-beta-induced epithelial to mesenchymal transition. Cell Res 19: 156-172, 2009.

29. Crighton D, Wilkinson S and Ryan KM: DRAM links autophagy to $\mathrm{p} 53$ and programmed cell death. Autophagy 3: 72-74, 2007.

30. Mah LY, O'Prey J, Baudot AD, Hoekstra A and Ryan KM: DRAM-1 encodes multiple isoforms that regulate autophagy. Autophagy 8: 18-28, 2012.

31. Macintosh RL, Timpson P, Thorburn J, Anderson KI, Thorburn A and Ryan KM: Ryan, Inhibition of autophagy impairs tumor cell invasion in an organotypic model. Cell Cycle 11: 2022-2029, 2012.

32. Zhan Z, Xie X, Cao H, Zhou X, Zhang XD, Fan H and Liu Z: Autophagy facilitates TLR4- and TLR3-triggered migration and invasion of lung cancer cells through the promotion of TRAF6 ubiquitination. Autophagy 10: 257-268, 2014.

33. Liu S, Xie F, Wang H, Liu Z, Liu X, Sun L and Niu Z: Ubenimex inhibits cell proliferation, migration and invasion in renal cell carcinoma: The effect is autophagy-associated. Oncol Rep 33: 1372-1380, 2015.

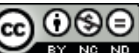

This work is licensed under a Creative Commons Attribution-NonCommercial-NoDerivatives 4.0 International (CC BY-NC-ND 4.0) License. 